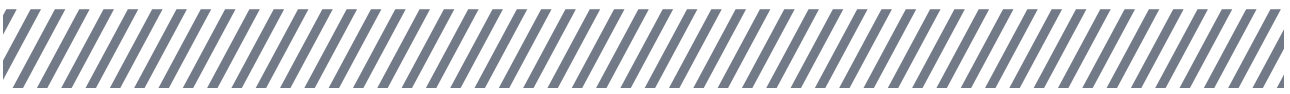

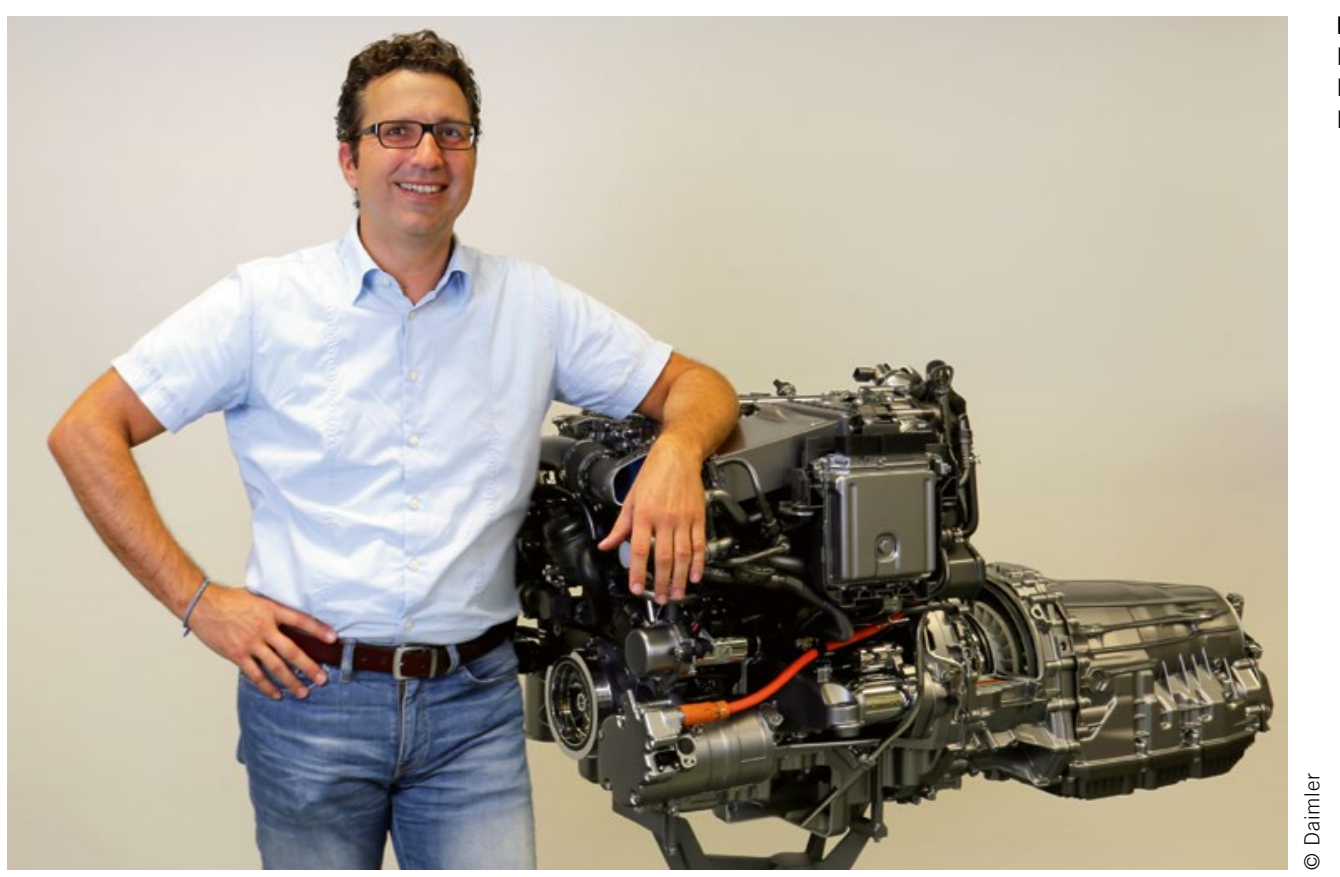

Dr. Ralph Weller

Leiter Berechnung und Benchmark Powertrain,

Daimler AG

\section{Digitale Systementwicklung moderner Pkw-Antriebe}

Die Zukunft des Antriebsstrangs vorherzusagen, ist schwierig. Um mögliche Szenarien zeitnah und in entsprechender Qualität und Performance verwirklichen zu können, wird es immer wichtiger, das gesamte Spektrum vom klassischen Verbrennungsmotor, über Hybride und E-Antriebe bis hin zur Brennstoffzelle zu entwickeln und technologisch abzusichern. Ein Hybridantriebsstrang mit mehreren kommunizierenden Steuergeräten erzeugt deutlich komplexere Zusammenhänge als ein klassischer Antriebsstrang. Diese komplexen Systeme gilt es ganzheitlich simulatorisch darzustellen, zu verstehen und auf modernsten Prüfständen zu beherrschen. Das Systemdenken wird dabei immer wichtiger.

Hierbei rückt der Einsatz von leistungsfähigen Berechnungstools mehr in den Fokus. Hinsichtlich der steigenden Komplexität moderner Powertrains durch die zunehmende Elektrifizierung sind herkömmliche, stark hardwareorientierte Entwicklungsansätze nicht mehr zielführend. Zur Beherrschung und Optimierung solcher komplexen Systeme sind zunehmend digitale Entwicklungsmethoden notwendig, ohne die eine effiziente Entwicklung hinsichtlich Kosten und Zeit nur schwer möglich wäre. Hierzu wurden im Hause Daimler leistungsfähige und prognosesichere Berechnungsmethoden entwickelt, die dazu geführt haben, den frühen Entwicklungsprozess rein digital zu gestalten. Die enge Vernetzung zwischen Konstruktion, Simu- lation und Einbindung von Ergebnissen früherer Powertrains ermöglicht die Entstehung einer digitalen Baustufe anstatt einer kostenintensiven ersten Hardware-Charge.

Der aktuelle Wandel vom konventionellen zum elektrifizierten Powertrain ist ein maßgeblicher Komplexitätstreiber, der bereits in der Auslegungs- und Konzeptphase beherrscht werden muss. Daher setzt Daimler bereits in der frühen digitalen Konzeptentwicklung stark auf den Einsatz von Software in the Loop-Ansätzen (SiL). Diese basieren auf der Kopplung von Steuergerätemodellen mit physikalischen Streckenmodellen und sind charakterisiert durch eine hohe Prognosefähigkeit. Die eingesetzte, modular aufgebaute SiL-Entwicklungsplattform ermöglicht den einfachen Austausch von komplexen und daher nicht echtzeitfähigen Modellen durch Real-time-Modelle, sodass der „Prognose-SiL“ zum „Applikations-SiL“ weiterentwickelt werden kann (durchgängige Toolkette), mit dem Ziel, diesen zur Offline-Bedatung in der Applikation einzusetzen.

Die konsequente Optimierung von Bauteilen und die perfekte Auslegung des Gesamtsystems in der digitalen Konzeptphase sind somit Voraussetzung, um bei steigender Komplexität und begrenzten Ressourcen einen in allen Details perfekten Powertrain zu erschaffen mit dem Ziel, in Sachen Verbrauch, Emissionen, Performance, Zuverlässigkeit und NVH aber auch bezüglich Wirtschaftlichkeit und Flexibilität neue Maßstäbe zu setzen. 